Original Article

\title{
EVALUATION OF ANTIM ICROBIAL AND CYTOTOXIC ACTIVITY OF ELECTRON BEAM IRRADIATED ENDODONTIC SEALER
}

\author{
A Geethashri', KJ Palaksha ${ }^{2}$, B M ohana Kumar ${ }^{2}$, KR Sridhar ${ }^{3}$, Ganesh Sanjeev $^{4}$, A Veena Shetty ${ }^{5}$ \\ ${ }^{1}$ Senior Research Fellow, Nitte University Centre for Science Education \& Research, ${ }^{2}$ Associate Professor, Nitte \\ University Centre for Stem Cell Research \& Regenerative Medicine, ${ }^{5}$ Associate Professor, Department of M icrobiology, \\ K.S. Hegde M edical Academy, Nitte University, ${ }^{3}$ Professor \& HOD, Department of Biosciences, ${ }^{4}$ Senior Physicist, \\ Mangalore University, Mangalore, India.

\section{Correspondence: \\ Veena A Shetty} \\ Associate Professor, Department of M icrobiology, K.S. Hegde M edical Academy, Nitte University, M angalore - 575 018, India. \\ Email: vndshetty@yahoo.co.in
}

\begin{abstract}
:
Background : The persistent pathogenic microorganisms in root canal system even after chemo-mechanical preparations cause endodontic infection and failure of the treatment. Thus the filling material, in addition to its good sealing ability, should offer long term antimicrobial effect and be non-toxic to cells and dentin. Zinc Oxide Eugenol (ZOE) is the most commonly used root canal sealer in endodontics. Electron beam (e-beam) is an ionizing radiation and known to cause physiochemical and biological changes. The aim of this study was to evaluate the effect of e-beam irradiation on bioactive properties of ZOE.
\end{abstract}

Methodology : The homogenous mixture of ZOE was prepared as per manufacturer's instructions and discs of $6 \mathrm{~mm}$ were prepared by loading the paste into sterile moulds. After complete drying discs were aseptically removed and subjected to e-beam irradiation at doses of $250 \mathrm{~Gy}, 500 \mathrm{~Gy}, 750 \mathrm{~Gy}$ and $1000 \mathrm{~Gy}$ at M icrotron Centre, M angalore University. Antimicrobial and antibiofilm properties of both control(non-irradiated) and irradiated sealer against Enterococcus faecalis, Staphylococcus aureus, Streptococcus mutans and Candida albicans were determined by well diffusion method and antibiofilm by O'Toole method, respectively. The cytotoxicity was determined by using $\mathrm{M} T \mathrm{~T}$ assay on human gingival fibroblasts.

Results : The antimicrobial effect of ZOE was observed only against S. aureus and C. albicans. The ZOE sealer irradiated at 1000 Gy showed a significantly $(P<0.001)$ increased antimicrobial effect against $\mathrm{S}$. aureus and $\mathrm{C}$. albicans compared to control ZOE. However, the substantially increased antibiofilm activity against C. albicans was noticed in the ZOE irradiated at $250 \mathrm{~Gy}$. There was no significant $(P>0.05)$ difference in cytotoxicity between control and irradiated ZOE.

Conclusion : The e-beam irradiated endodontic sealer ZOE at 1000 Gy and 250 Gy significantly enhanced the antimicrobial and antibiofilm activity respectively without changing its biocompatibility.

Keywords : Electron beam irradiation, Endodontic sealers, Zinc Oxide Eugenol, Oral pathogens, Antimicrobial activity, Cytotoxicity.

\section{Introduction:}

The outcome of endodontic treatment depends on the complete elimination of microorganisms present in the root canal by instrumentation, irrigation and the use of effective sealant ${ }^{1}$. An ideal root canal sealer should prevent

\begin{tabular}{|c|}
\hline Access this article online \\
\hline Quick Response Code \\
\hline
\end{tabular}

and recontamination of the canal system completely and be non a ntigenic, nontumerogenic and nontoxic ${ }^{2}$.
Several materials have been recommended as root sealers however, none of them has so far been proved to be an ideal sealer ${ }^{3}$. Despite differences of opinions on the spectrum of antimicrobial activity, Zinc Oxide Eugenol (ZOE) is one of the most commonly used endodontic sealersin root canal treatment ${ }^{4 \cdot 6}$.
E-beam radiation is a form of ionizing energy has been introduced as a means of sterilizing single-use, disposable health care products. Ionizing radiation induces structural changes in the native pharmaceutical compound resulting in altered physico-chemical, microbiological and toxicological properties ${ }^{7}$. It has also been reported to be an 
effective tool to decompose the organic substances and reduce the toxicity ${ }^{8}$.

Enterococcus faecalis, Staphylococcus aureus, Streptococcus mutans and Candida albicans are among the few endodontic pathogens causing the failure of the root canal treatment and reinfections ${ }^{9},{ }^{10}$. In this study, antimicrobial and cytotoxic activity of the most commonly used sealer ZOE was evaluated before and after exposing to e-beam irradiation against persistent microbes and human gingival fibroblasts, respectively.

\section{M aterials and Methods:}

The commercially available ZOE (Septodent, M umbai) was purchased from the market. The emulsion of zinc oxide and eugenol prepared as per manufacturers' instructions. The discs of ZOE $(6 \times 4 \mathrm{~mm})$ after complete drying, were aseptically removed from the mould and divided into control and experimental (irradiated) groups. The experimental group discs were irradiated by e-beam at 250 Gy, 500 Gy, 750 Gy and 1000 Gy at a dose rate of 500 Gy/min using the Microtron facility available at Department of Physics, M angalore University.

The antimicrobial activity of control and irradiated sealers was evaluated by well diffusion and biofilm inhibition assays. The microorganisms E. faecalis (ATCC 29212), S. aureus (ATCC 25923), S. mutans (M TCC 890) and C. albicans (ATCC 90028) were obtained from the stock cultures of Nitte University Centre for Science Education and Research. The bacteria and fungus were subcultured in Mueller Hinton Agar (M HA) and Sabouraud Dextrose Agar (SDA), respectively.

The well diffusion assay was carried out according to the slightly modified method of Filho MT et al ${ }^{11}$. Briefly, the optical densities of seven hrs old bacterial and fungal cultures were adjusted to $0.5 \mathrm{McFarland}$ standard and swabbed uniformly on the $20 \mathrm{ml}$ of solidified M HA and SDA medium. Then, control and irradiated sealers were placed aseptically to the wells of $6 \times 4 \mathrm{~mm}$ prepared by using sterile cork and borer. Followed by overnight incubation of culture plates at $37^{\circ} \mathrm{C}$, the zones of inhibition were recorded in $\mathrm{mm}$.

The biofilm inhibition ability of sealer was determined on the endodontic pathogens grown in 96 well plates as described by O'Toole ${ }^{12}$. The control and irradiated sealers were immersed in $1 \mathrm{ml}$ of Mueller Hinton Broth (M HB) and Sabouraud Dextrose Broth (SDB) for 24 hrs at room temperature. The $20 \mu \mathrm{l}$ of the elute was inoculated into the each wells and incubated for $15 \mathrm{~min}$ at room temperature. Then the contents were discarded, followed by gentle wash in phosphate buffered saline (PBS, pH 7), the biofilms were stained with $0.1 \%$ crystal violet stain for $15 \mathrm{~min}$. Excess stain was removed by washing the biofilm in double distilled water for three times. Following the destaining with $30 \%$ acetic acid, the quantification of viable cells was performed by taking the optical density reading at $560 \mathrm{~nm}$ using Lisa chem plate reader.

The cytotoxicity of sealers to human gingival fibroblasts was evaluated by MTT 3-(4,5-dimethythiazol-2-yl)-2,5diphenyl tetrazolium bromide assay ${ }^{13}$. Briefly, $20 \mu \mathrm{l}$ of eluted fraction of sealers, which were pre-incubated in $1 \mathrm{ml}$ Dulbecco's M odified Eagle Medium (DM EM) for $24 \mathrm{hrs}$ at room temperature was inoculated to well containing $1 \times 10^{4}$ cells in 96-well microtiter plate and incubated for 15 min at $37^{\circ} \mathrm{C}$ in $5 \% \mathrm{CO}_{2}$ incubator. Then the wells were washed once with PBS. The cytotoxicity of sealers was evaluated by incubating the cells with $100 \mu$ l of $\mathrm{M} \mathrm{TT}$ dye $(0.05 \mathrm{mg} / \mathrm{ml})$ in PBS for 4 hrs at $37^{\circ} \mathrm{C}$ in $5 \% \mathrm{CO}_{2}$ incubator. The intensity of the colour was measured by adding dimethyl sulphoxide (DM SO) at $545 \mathrm{~nm}$ using Lisa chem plate reader.

All the assays were performed in triplicates. The results obtained were analyzed by one way ANOVA. The level of significance was considered at $5 \%$.

\section{Resultsand Discussion:}

The antimicrobial activity of ZOE was observed only against S. aureus and C. albicans. However, the ZOE sealer irradiated at 1000 Gy showed a significantly $(P<0.001)$ increased antimicrobial activity against $\mathrm{S}$. aureus (Figure 1 and 7) and C. albicans (Figure 2 and 8 ) compared to control ZOE. 


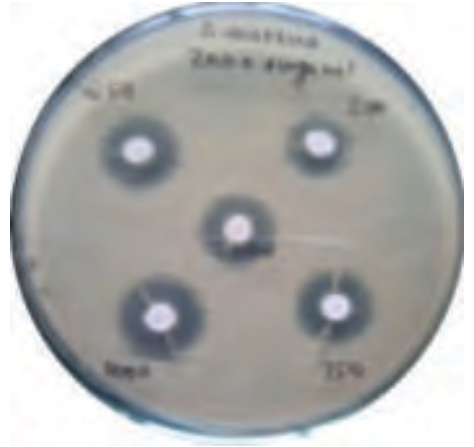

Figure 1 : Zone of inhibition by control and irradiated ZOE against S. aureus

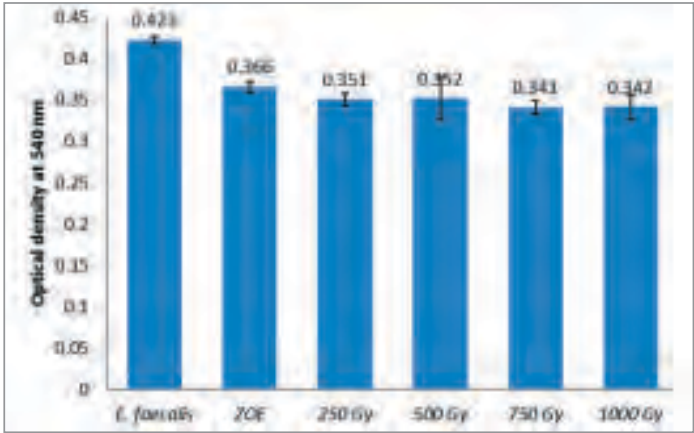

Figure 3 : E. faecalis biofilm suppression by ZOE and irradiated ZOE.

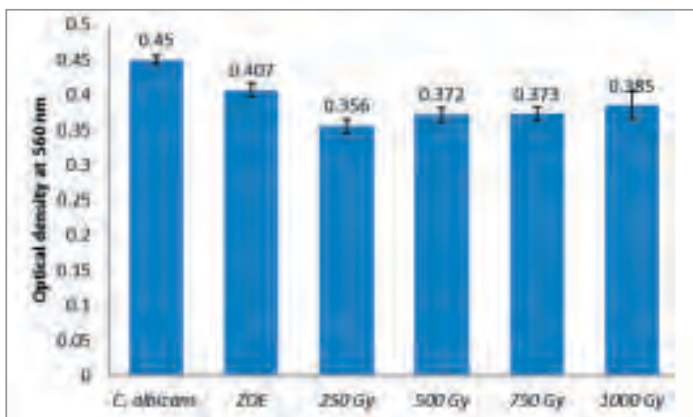

Figure 5 : C. albicans biofilm suppression by ZOE and irradiated ZOE.

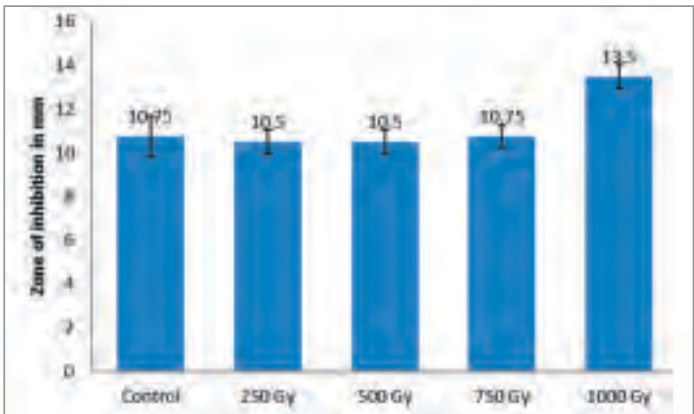

Figure 7: The antimicrobial activity of ZOE at 1000 Gy against S. aureus

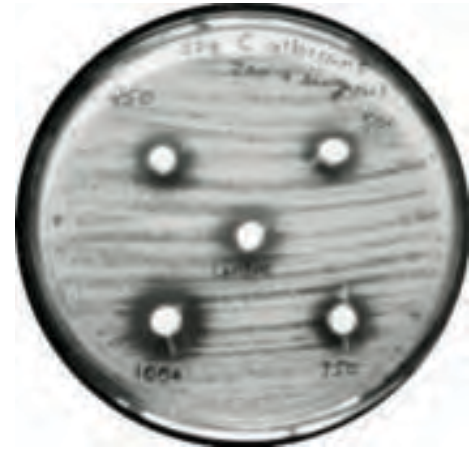

Figure 2 : Zone of inhibition by control and irradiated ZOE against C. albicans

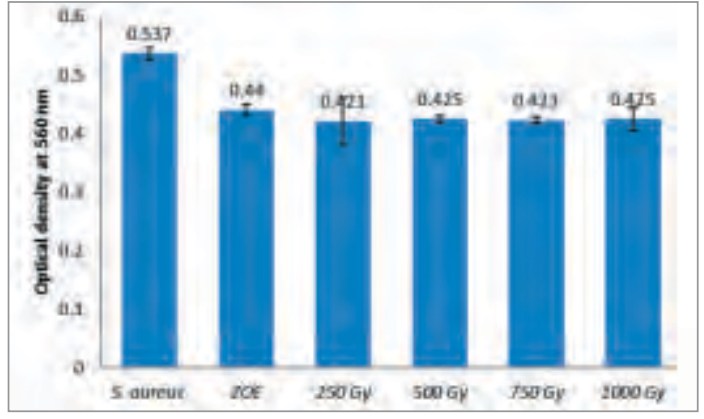

Figure 4: S. aureus biofilm suppression by ZOE and irradiated ZOE.

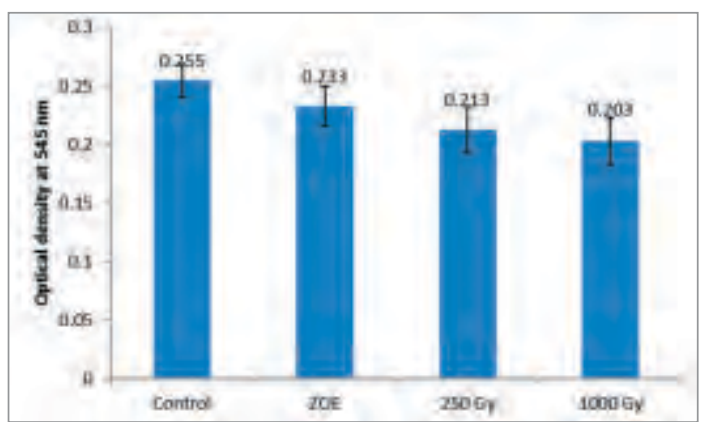

Figure 6: Cytotoxicity of ZOE and irradiated ZOE

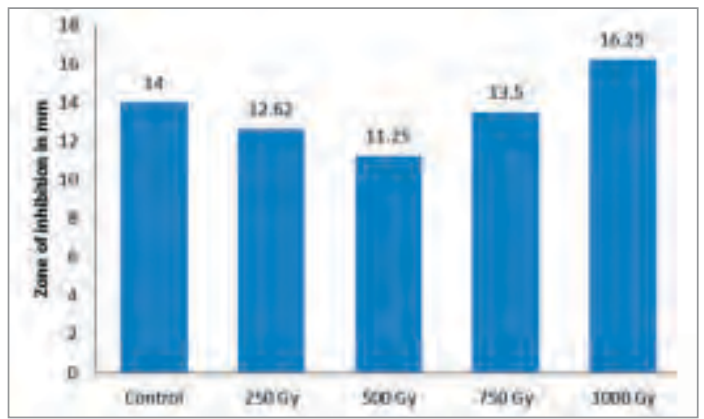

Figure 8 : The antimicrobial activity of ZOE at 1000 Gy against C. albicans 
The antibiofilm activity of sealer was observed against $\mathrm{E}$. faecalis, S. aureus and C. albicans. In addition, the irradiated sealers showed an increased inhibition of $\mathrm{E}$. faecalis (Figure 3), S. aureus (Figure 4) and C. albicans (Figure 5) biofilm. The ZOE irradiated at 250 Gy showed the substantially increased $(P<0.01)$ suppression effect on the formation of biofilm by C. albicans. In cytotoxicity assay, the percentage viability of cells was observed as $92.82 \%$, $83.52 \%$ and $87.12 \%$ when treated with control, $250 \mathrm{~Gy}$ and 1000 Gy irradiated ZOEs, respectively, and demonstrated that the cytotoxic effect of irradiated ZOEs was insignificant ( $P>0.05)$ compared to control (Figure 6).

Zinc oxide eugenol based sealers are most commonly employed sealants in endodontics ${ }^{14}$. Eugenol is a potent antimicrobial agent, therefore the antimicrobial activity of ZOE- based sealants attributed to the free eugenol released from set material ${ }^{15}$. Supporting these observations, previous studies also reported that the cytotoxic effect of ZOE-based sealants due to their free eugenol component ${ }^{16}$.

It has been reported that the irradiation of pharmaceutical compounds did not affect the biological properties like antimicrobial activity ${ }^{7,17}$. In addition, it is used as a tool to degrade the detergents and reduce the adverse effects. The significantly decreased acute toxicity of sodium

\section{References:}

1. Sandqvist G. Ecology of the root canal flora.J Endod 1992;18:427-430.

2. D'Souza L Henston, Sharma N, Chander S, Singh S, D'Souza R. Root canal sealers and its role in successful endodontics-A review. Annals of Dental Research 2012;2(2):68-78.

3. Zarrabi $\mathbf{M H}$, Javidi $M$, Naderinasab $M$, Gharechahi $M$. comparative evaluation of antimicrobial activity of three cements: new endodontic cement (NEC), mineral trioxide aggregate (M TA) and portland. Journal of Oral Science 2009;51(3):437-442.

4. Gomes BPFA, Pedroso JA, Jacinto RC, Visnns M E, Ferraz CCR, Zaia AA, In vitro Evaluation of the Antimicrobial Activity of Five Root Canal Sealers. Braz Dent J 2004;15(1):30-35.

5. Queiroz AM, Nelson-Filho P, Silva LAB, Assed S, Silva RAB, ITO IY. Antimicrobial Activity of Root Canal Filling M aterials for Primary Teeth: Zinc oxide and eugenol Cement, Calen Paste Thickened with Zinc Oxide, Sealapexand EndoREZ. Braz DentJ 2009;20(4):290-296.

6. Pizzo G, Giammanco GM, Cumbo E, Nicolosi G, Gallina G. In vitro antimicrobial activity of endodontic sealers. Jornal of Dentistry 2006;34:35-40.

7. Beteshobabrud R, Nabardi $F$. The stability studies of penicillin and ampicillin following ?-irradiation in the solid state. Iranian Journal of Pharmaceutical Research 2009;8(3):153-157.

8. Romanelli M F, M oraes M CF, Villavicencio ALCH, Borrely SI. Evaluation of toxicity reduction of sodium dodecyl sulfate submitted to electron dodecyl sulfate was seen after e-beam irradiation at 3 and 6 $\mathrm{kGy}^{8}$. So in this study, efforts have been made to evaluate the effect of e-beam on antimicrobial and cytotoxic activity on locally available ZOE-based sealer.

In this study the antimicrobial activity of ZOE-based sealant was observed only against $\mathrm{S}$. aureus and $\mathrm{C}$. albicans. On both the microbes, a significantly increased antimicrobial activity of irradiated ZOE- based sealer was seen at $1000 \mathrm{~Gy}$ of e-beam irradiation. Biofilm of S. aureus, E. faecalis and C. albicans was significantly suppressed by ZOE. There was no significant difference in suppression of $\mathrm{E}$. faecalis and $\mathrm{S}$. aureus biofilm by irradiated ZOE, but C. albicans biofilm was significantly suppressed by ZOE irradiated at $250 \mathrm{~Gy}$. The biocompatibility of ZOE was not altered as there was no significant $(P>0.05)$ difference between $Z O E$ and irradiated ZOE at $250 \mathrm{~Gy}$.

\section{Conclusions:}

Based on the present study e-beam irradiation might be a tool in enhancing the antibacterial and antifungal activity of the sealers.

\section{Acknowledgement:}

Authors gratefully acknowledge the financial support given by the Department of Atomic Energy, Board of Research in Nuclear Sciences (BRNS), Government of India.

beam irradiation. Radiation Phys. Chem. 2004:71;409-411

9. Narayanan LL, Vaiahnavi C. Endodontic M icrobiology. J Conserv Dent 2010;13(2):233-239.

10. Peciuliene V, Reynaud AH, Balciuniene I, Haapasalo M. Isolation of yeasts and enteric bacteria in root-filled teeth with chronic and apical periodontitis. Int Endod J 2001;34(6):429-34.

11. Filho MT, Tonomaru JMG, Barros DB, Watanabe E, Ito IY. In vitro antimicrobial activity of endodontic sealers, M TA-based cements and Portland cement. J Oral Sci. 2007:49:41-45.

12. O'Toole G.A. M icrotiter Dish Biofilm Formation Assay. J Vis Exp. 2011; 30(47):1-2.

13. Sergeant JM , Taylor CG. Appraisal of the MTT assay as a rapid test of chemosensitivity in acute myeloid leukaemia. Br. J.Cancer 1989; 60:206-210

14. Kaiwar A, Nadig G, Hegde J, Lekha S. Assessment of antimicroibial sealers on Enterococcu faecalis: An in vitro study. World Journal of Dentistry 2012;13(1):26-31.

15. Hume WR. The pharmacological and toxicological properties of zinc oxide- eugenol. Journal of American Dental Association 1986:113:789-91.

16. Lindqvist $\mathrm{L}$, Otteskog 0 . Eugenol- liberation from the dental materials and effect on human diploid fibroblast cells. Scandinavian Journal of Dental Research 1981;89:552-556. 
17. Singh B, Parwate DV, Shukla SK. Radiosterelization of Fluroquinolones and Cephalosporines: Assessment of radiation Damage on Antibiotics by changes in optical property and colorimetric parameters AAPS PharmSciTech 2009;10(1):34-43. 